sciendo

The Impossibility of Temporal Relations Between Non-identical

Times: New Arguments for Presentism

\author{
Jeffrey Grupp \\ Western Michigan University
}

Disputatio Vol. 1, No. 18

May 2005

DOI: $10.2478 /$ disp-2005-0001

ISSN: 0873-626X

(C) 2005 Grupp. Creative Commons Attribution-NonCommercial-NoDerivs 3.0 License 


\title{
The impossibility of temporal relations between non-identical times: new arguments for presentism
}

\author{
Jeffrey Grupp \\ Western Michigan University
}

\begin{abstract}
I argue that relations between non-identical times, such as the relations, earlier than, later than, or 10 seconds apart, involve contradiction, and only co-temporal relations are non-contradictory, which would leave presentism the only non-contradictory theory of time. The arguments I present are arguments that I have not seen in the literature.
\end{abstract}

\section{Introduction}

In this paper I attack the non-presentist position in the philosophy of time that there are relations that (are alleged to) connect any nonidentical times. In this introduction I will discuss these temporal relations that many philosophers and physicists allege exist, and in sections 2 and 3 I give novel arguments for their nonexistence.

I only discuss the temporal relations that many philosophers and physicists tell us connect non-identical times. Examples of such temporal relations are the relations, earlier than, parthood, and 10 seconds apart. I do not discuss relations that a time may have with itself, such as simultaneity. I only argue that if any times are not identical, they cannot share temporal relations. ${ }^{1}$ By 'non-identical times,' I am

\footnotetext{
${ }^{1}$ I will also briefly argue in subsection 2.4 below against all varieties of monadic relatedness possessed by temporal objects or times. Campbell discusses this position: 'Monadists propose to replace the relational $a \mathrm{R} b$ with two monadic propositions, $\mathrm{F} a$ and Gb, which attribute qualities to $a$ and $b$ individually.' (Campbell 1990, 102) Monadic relatedness is given in terms of monadic facts: $t_{1}$ 's relatedness to $t_{2}$, where relatedness is, for example, a monadic property of $\mathrm{t}_{1}$, not a shared polyadic property coexemplified with $t_{2}$. Monadic relatedness does not exist temporally between $t_{1}$ and $t_{2}$. And $t_{1}$ 's non-platonistic monadic property, related to $t_{2}$, is not located where $t_{2}$ is, but only where $t_{1}$ is. My arguments in subsection 2.4 specifically focus on monadic
}

Disputatio, Vol. I, No. 18, May 2005 
denoting times that (allegedly) share temporal relations such as, temporal distance, non-simultaneity, not identical to, and so on. (The temporal relations between non-identical times, are, along with the times, typically considered to be constituents of time.) If relations between times do not exist, then times are not before or after one another, and if that is the case all times would coincide, which would indicate that there is only one time, and thus presentism would be the correct theory.

Throughout this paper I will refer to divisible (non-basic) temporal extensions as 'durations.' Non-basic durations are typically considered to be either infinitely divisible, or to be composed of basic building blocks. (Some may deem that any duration is divisible, and so I only need to use the expression 'duration,' rather than the expressions 'divisible duration' or 'non-basic duration.' But I will distinguish between divisible and indivisible durations, since many physicists, especially some quantum gravity theorists, hold that a Planck time is a basic building block of time that has a temporal size (a duration).) Regardless of which is the correct position - regardless of whether or not durations are infinitely divisible (i.e., durations are not composed of time points, Plank times, or any sort of time atoms), or involve basic building blocks of time - both positions involve relations between non-identical times.

In this paper, I will refer to both basic times and durations (divisible or indivisible durations) as 'times.' For example, one year is a time, one hour is a time, one nanosecond is a time, and one basic building block of time is a time. I will call the relata (non-identical times) that are connected by temporal relations $t_{1}, t_{2}$, and $t_{3}$. In the examples I give in this paper, I will often refer only to $t_{1}$ and $t_{2}$, and only occasionally refer to three times, $t_{1}, t_{2}$, and $t_{3}$. The examples of $t_{1}$ and $t_{2} I$ will use in this paper are two basic times that are 10 seconds apart, or a duration that might be a part of another duration $\left(t_{1}=\right.$ one minute, $\mathrm{t}_{2}=$ one hour).

In sections 2 and 3, I will argue that there is a specific problem to do with any variety of a temporal relation between or among any non-

relatedness. In this paper, I will mainly discuss relations, since monadic relatedness has been discussed far less in the literature since Russell's Principles of Mathematics, where relations were argued to be irreducible. (One philosopher who does discuss monadic relatedness at length is Keith Campbell.) I will however refer to both relations and monadic relatedness at various places in the paper, but I will mainly be concerned with relations hereafter, only infrequently mentioning monadic relatedness. 
identical times (between or among $t_{1}$ and $t_{2}$ ): temporal relations between $t_{1}$ and $t_{2}$ apparently cannot be located in time, $T$ ( $I$ will call temporal relations that are located in time non-platonistic temporal relations), and they also cannot be timeless, $\sim \mathrm{T}$ (I will call temporal relations that are not located in time platonistic temporal relations). If temporal relations between or among $t_{1}$ and $t_{2}$ are neither nonplatonistic $(\mathrm{T})$ nor platonistic $(\sim \mathrm{T})$, they apparently involve contradiction, since they would be describable as $\sim(\mathrm{T} \vee \sim \mathrm{T})$, which translates to $\sim \mathrm{T} \wedge \mathrm{T}$. In section 2 I discuss hitherto unnoticed problems to do with non-platonistic temporal relations, $T$. If my reasoning is correct, only platonistic temporal relations, ${ }^{2} \sim \mathrm{T}$, could be considered to exist among $t_{1}$ and $t_{2}$. In section 3 I consider platonistic temporal relations among $t_{1}$ and $t_{2}$, where $I$ also come to serious problems when considering them.

\section{Non-platonistic relations between non-identical times}

In this section I discuss apparent problems to do with non-platonistic temporal relations between $t_{1}$ and $t_{2}$. In subsections 2.1 and 2.2, I discuss problems to do with noncomplex non-platonistic temporal relations between $\mathrm{t}_{1}$ and $\mathrm{t}_{2}{ }^{3}$ In subsection 2.3 I discuss problems to do with specific sorts of complex non-platonistic temporal relations that are not affected by the reasoning against non-platonistic noncomplex temporal relations between or among $\mathrm{t}_{1}$ and $\mathrm{t}_{2}$ given in subsections 2.1 and 2.2. In subsection 2.4 I discuss a problem to do with nonplatonistic temporal monadic relatedness.

\footnotetext{
${ }^{2}$ It is standard to consider platonistic relations as those which are not in the spatiotemporal world, whereas non-platonistic relations are not outside of the spatiotemporal world, as Loux discusses:
}

What are the issues separating the Aristotelian realists from Platonists? ... Aristotelians typically tell us that to endorse Platonic realism is to deny that properties, kinds, and relations, need to be anchored in the spatiotemporal world. As they see it, the Platonist's universals are ontological 'free floaters' with existence conditions that are independent of the concrete world of space and time. But to adopt this conception of universals, Aristotelians insist, is to embrace a two-worlds' ontology... On this view, we have a radical bifurcation of reality, with universals and concrete particulars occupying separate and unrelated realms... [T] here [is a] connection between spatiotemporal objects and beings completely outside of space and time. (Loux 1998, 46)

${ }^{3}$ Noncomplex relations are simple (partless), and complex relations are not simple. 


\subsection{Noncomplex temporal relations of non-zero temporal size}

It appears that there are two ways to conceptualize a non-platonistic temporal relation, if the relation (allegedly) connects $t_{1}$ and $t_{2}$.

1. A non-platonistic relation is temporally extended between $t_{1}$ and $t_{2}$. $I$ will discuss varieties of this sort of non-platonistic temporal relation primarily in this subsection, but also in parts of other subsections of this section. ${ }^{4}$

2. The second way to conceptualize non-platonistic temporal relations between $t_{1}$ and $t_{2}$ is by considering them as not temporally extended between $t_{1}$ and $t_{2}$, but only temporally located where $t_{1}$ and $t_{2}$ are. Such temporal relations are in time, but are temporally unextended (durationless) entities. ${ }^{5}$ I discuss this position primarily in subsection 2.2, but also in parts of other subsections.

In this subsection, I discuss temporally extended non-platonistic temporal relations between $t_{1}$ and $t_{2}$. In other words, I am only considering temporal relations of non-zero temporal size that connect at least two non-identical temporal locations: relations of non-zero temporal size that connect $t_{1}$ and $t_{2}$, where $t_{1} \neq t_{2}$.

It is not uncommon for philosophers to hold that non-platonistic temporal relations, in addition to the times that make up time, are not occupants of time, but rather contribute to the makeup of time, without being occupants of time. In this subsection I will argue that nonplatonistic temporal relations that are constituents of time, if they are any variety of non-platonistic temporal relation (temporally extended, temporally unextended, etc.), can only be temporally located: they only can be occupants of time. I do this next in 2.1.1. In 2.1.2 I give an argument that leads to the conclusion that temporally extended non-platonistic temporal relations between non-identical times are contradictory. In 2.1.3 I will consider an objection to the argument given in 2.1.2.

\footnotetext{
${ }^{4}$ It is this 'betweenness,' where relations are not merely at the locations of their relata, that monadists often reject about relations.

${ }^{5}$ When I refer to properties as 'entities,' I use the word 'entity' in the broadest possible sense, and in the way that many other metaphysicians refer to n-adic properties as 'entities.' (For example, Esfeld $(2003,10)$, Lowe $(2002,16)$, Moreland (2001, 13), and many others. Also, a passage from Reinhardt Grossmann at the very start of section 3 below involves Grossmann referring to 'abstract qualities' as 'entities.' (Grossmann, 1990, 7))
} 


\subsubsection{Non-platonistic temporal relations can only be occupants of time}

I will next argue that non-platonistic temporal relations between $t_{1}$ and $t_{2}$ can only be considered as occupants of time. This is relevant to my reasoning in 2.1.2 where I argue that non-platonistic temporally extended temporal relations between $t_{1}$ and $t_{2}$ are contradictory.

If there are non-platonistic temporal relations that contribute to the makeup of time, since they interrelate non-identical times, the nonplatonistic relations must coincide with those times $\left(t_{1}\right.$ and $\left.t_{2}\right)$ that they interrelate. Further, the temporal relation must coincide with the entirety of the time it coincides with, regardless of whether or not the interrelated times are basic building blocks of time or durations (divisible or indivisible durations). If the temporal relation only coincided with a part of one of the times it relates, then statements such as ' $t_{1}$ is related to $t_{2}$ ' would be false, since only parts of $t_{1}$ or $t_{2}$ would take part in the co-exemplification of the non-platonistic relation (and instead, statements such as, for example, ' $t_{1}$ is related to part of $t_{2}$ ' would be true). For example, if one hour $\left(t_{1}\right)$ is related to one minute $\left(t_{2}\right)$ by the temporal relation parthood, it can only be the case that the entire hour coincides with the temporal relation in order for the hour in question to be a relatum of the temporal relation, parthood. If only part of the hour coincided with the temporal relation, then the statement 'the minute is related to the hour' would be false, and the statement 'the minute is related to the forty-five minute duration' would be true, if, for example, the relation only coincided with three quarters of the hour. Similar reasoning holds for Planck basic building blocks of time. For example, it cannot be the case that, with respect to a Planck time, the relation just contacts the surface of, or a left side of, a single Planck unit of time. (Also, it is unclear that what has just been written about a Planck time is coherent, given that it is unclear if a 'side' or 'surface' of a Planck time can even be discussed at all, since 'side' and 'surface' may be references to parts of the Planck time, or aspects of the Planck time not identical to the entirety of the single Planck time, rather than to the entirely of the Planck unit of time, and this is not possible since there are no parts or aspects of a Planck time that are not identical to the entirety of the Planck time.) Of course, if a relation did not attach or link to its relata (where 'attach' and 'link' denote the special exemplification tie that holds relations to their relata ${ }^{6}$ ), then there would be a

\footnotetext{
${ }^{6}$ See Loux 1998, 38-41.
} 
discontinuity of some sort between the temporal relation and its relata (times), which is absurd, since the relations then would not attach or link to their relata, and thus they would be relations that do not interrelate their relata.

For reasons just given, non-platonistic temporal relations that are constituents in the makeup of time must coincide with the entirety of the times that they interrelate. Times are temporal locations, and thus are not located in time. I will next discuss that this means that nonplatonistic temporal relations cannot also be temporal locations, even though the temporal relations are constituents of time. If the temporal relations were also temporal locations, then times and the temporal relations that connect the times to one another would coincide (overlap), where these coinciding entities would each be temporal locations. This has obvious problems, however, since two temporal locations that temporally overlap or coincide are not at a distance from one another, and cannot each be temporal locations, unless they are identical. But this cannot be the case since a temporal relation must be distinct from its relata. This implies that if there are non-platonistic temporal relations between non-identical times, since the nonplatonistic temporal relations are in time but are not themselves temporal locations, then they could only be located at places in time, in order to avoid the problems just discussed. But if that is the case, then non-platonistic temporal relations that are constituents of time would be temporally located relations that occupy time (they are located in time). Hereafter, for reasons just given, I will only discuss nonplatonistic temporal relations of any sort (complex, noncomplex, etc.) as being occupants of time, regardless of the fact that they are (allegedly) constituents of time.

2.1.2 The impossibility of non-platonistic noncomplex temporally extended temporal relations between $t_{1}$ and $t_{2}$

I next give an argument against non-platonistic, temporally extended, noncomplex relations between non-identical times, $t_{1}$ and $t_{2}$. If temporally extended, noncomplex, non-platonistic relations between nonidentical times occupy at least two non-identical temporal locations, then they apparently involve contradiction, for the following reasons.

If a temporally extended temporal relation is partless (noncomplex), it is a single entity. If a temporally extended, noncomplex temporal relation is describable by a statement, then the entire temporal relation is describable by the statement. For example, the entire 
relation would be describable by the statements, 'located at temporal location $t_{1}$ ', and, 'located at temporal location $t_{2}$.' If the relation is located at $t_{2}$, and if $t_{1} \neq t_{2}$, then by being at $t_{2}$, the noncomplex nonplatonistic temporal relation is describable by the statement, 'not located at $t_{1}$.' This could be said of any non- $t_{1}$ location that the nonplatonistic noncomplex temporal relation occupies. If the temporal relation occupies more than two times, and for that reason is located at three temporal locations, $t_{1}, t_{2}$, and $t_{3}$, at locations $t_{2}$ and $t_{3}$ the temporal relation would be describable by the statement, "not located at $t_{1}$. These are, however, statements that reveal the nonexistence of the temporal relation: since the relation is one, partless entity, if it is 'located at $t_{1}$ ', and 'not located at $t_{1}$,' each of these statements must describe the entire noncomplex non-platonistic temporal relation, and that implies the entire relation would be describable by selfcontradictory conjunction of the above statements: 'located at $t_{1}$ and not located at $\mathrm{t}_{1}$.'

2.1.3 Temporally extended noncomplex temporal relations only located at entire temporal locations

In this subsection I discuss an objection to the reasoning given in 2.1.2 where non-platonistic noncomplex (simple) temporally extended temporal relations between $t_{1}$ and $t_{2}$ were found to involve contradiction if they occupy two or more temporal locations.

Philosophers who hold that temporal relations are temporally extended may assert that if a relation is located at a certain time $t_{2}$, this does not imply that it therefore does not also have the property of being located at some other time, $t_{1}$. Such philosophers may assert that non-platonistic temporal relations can be wholly located at two different times. ${ }^{7}$ In order for a philosopher to hold this position, she would merely need to avoid my reasoning above where I held that there are statements such as 'not at $\mathrm{t}_{1}$ ' that describe the temporal relation; she must hold that such statements do not describe noncomplex non-platonistic temporal relations between $t_{1}$ and $t_{2}$. This might be done by holding that the temporally extended temporal relation can only be considered at the entire time it is located is at. To hold this objection is to hold that in the previous subsection temporal relations

\footnotetext{
${ }^{7}$ I am grateful to a referee at Disputatio for making helpful comments that led me to discuss this objection. Around the same time, John Dilworth also expressed an objection to this that is very similar, and thus I am grateful to him for that.
} 
have been inaccurately described, since it may be the case that a temporally extended noncomplex non-platonistic relation might only be accurately described as being at its entire temporal location (call it $\mathrm{t}_{1} \mathrm{t}_{2} \mathrm{t}_{3}$ ), not at a part (sub-location) of its temporal location, such as the basic times, $t_{1}, t_{2}$, or $t_{3}$. According to this objection, the temporally extended non-platonistic temporal relation that connects $t_{1}$ and $t_{3}$, where $t_{2}$ is between $t_{1}$ and $t_{3}$, is not located at the basic times, $t_{1}, t_{2}$, and $t_{3}$, of the temporal locations, $t_{1} t_{2} t_{3}$. Rather, only the entirety of $t_{1} t_{2} t_{3}$ an be called the noncomplex, non-platonistic, temporally extended temporal relation's location. On this scenario, the statement,

'The noncomplex non-platonistic temporal relation between $t_{1}$ and $t_{3}$ is located at temporal location $\mathrm{t}_{1} \mathrm{t}_{2} \mathrm{t}_{3}$,

is true, and statements about the temporal relation being at any nonbasic sub-location of $t_{1} t_{2} t_{3}$ (i.e., sub-location $t_{1} t_{2}$ or sub-location $t_{2} t_{3}$ ), or at the individual basic sub-locations, of $\mathrm{t}_{1} \mathrm{t}_{2} \mathrm{t}_{3}$, are all false, such as the statements,

'The noncomplex non-platonistic temporal relation between $t_{1}$ and $t_{3}$ is located at $\mathrm{t}_{1}$,'

'The noncomplex non-platonistic temporal relation between $t_{1}$ and $t_{3}$ is located at $\mathrm{t}_{2}$, or

'The noncomplex non-platonistic temporal relation between $t_{1}$ and $t_{3}$ is located at $\mathrm{t}_{3}$,

In this subsection, I will argue that this objection fails. According to this objection, the temporally extended, noncomplex, non-platonistic temporal relation is at temporal location $t_{1} t_{2} t_{3}$, but aspects of the relation at $t_{1}, t_{2}$, or $t_{3}$ cannot be discussed, since there are no such aspects of the temporal relation that are not identical to the whole relation. Nevertheless, since the relation extends temporally between $t_{1}$ and $t_{3}$, it is important to note that all of the individual basic times, $t_{1}, t_{2}$, or $t_{3}$, can only be occupied by something to do with the temporal relation. By this I merely mean that when we consider the sublocations of $t_{1} t_{2} t_{3}$ and when we ask the question of whether or not the sub-locations $\left(t_{1}, t_{2}, t_{3}\right)$ of the entire temporal location $\left(t_{1} t_{2} t_{3}\right)$ are occupied, we apparently can only conclude that they are not unoccupied with respect to the temporal relation. The reason that $t_{1}, t_{2}$, or $t_{3}$ must 
be occupied by something to do with the temporal relation is because the entire temporal location, $\mathrm{t}_{1} \mathrm{t}_{2} \mathrm{t}_{3}$, that the non-platonistic temporal relation is at, is a time that is made up of more fundamental temporal locations, and if the temporal relation is at a non-basic temporal location (such as $t_{1} t_{2} t_{3}$ ) and accordingly occupies the entire temporal location, it must also be the case that the temporal relation occupying $\mathrm{t}_{1} \mathrm{t}_{2} \mathrm{t}_{3}$ leads to each of the temporal locations that make up $\mathrm{t}_{1} \mathrm{t}_{2} \mathrm{t}_{3}$ also being occupied.

A temporal location would not be occupied at all if none of its sublocations that compose it were occupied. Put in slightly different words, if a temporal relation occupying a temporal location $\left(\mathrm{t}_{1} \mathrm{t}_{2} \mathrm{t}_{3}\right)$ does not occupy the more fundamental temporal locations $\left(t_{1} t_{2}, t_{2} t_{3}\right)$, or any of the basic times $\left(t_{1}, t_{2}, t_{3}\right)$, of the temporal region $t_{1} t_{2} t_{3}$, then the temporal relation does not occupy the entire temporal location. For these reasons, the temporal relation's being at $t_{1} t_{2} t_{3}$ must also lead to all of the sub-locations of $t_{1} t_{2} t_{3}$ being occupied. But this poses a serious problem for the noncomplex, temporally extended, nonplatonistic temporal relation at temporal location $\mathrm{t}_{1} \mathrm{t}_{2} \mathrm{t}_{3}$ : if the relation can be described as occupying sub-locations of $\mathrm{t}_{1} \mathrm{t}_{2} \mathrm{t}_{3}$, the problems of the previous subsection ensue.

The reasoning about temporal locations just given, where nonbasic temporal locations were discussed as being composed of sublocations, and of basic sub-locations (if time is not infinitely divisible), is the case for any non-basic temporal location, since any non-basic temporal location is made up of more fundamental temporal locations. If it were the case that a non-basic temporal location, such as $\mathrm{t}_{1} \mathrm{t}_{2} \mathrm{t}_{3}$, were not made up of more fundamental, or basic, temporal locations, then an extended and non-basic temporal location would not be made up of anything, and it would not be a temporal location at all. For these reasons, a non-basic temporal location is composed of more fundamental temporal locations, or basic temporal locations, and a temporal relation's occupying a non-basic temporal location must accordingly result in the more fundamental temporal locations, or basic temporal locations, also being occupied. The noncomplex, temporally extended, non-platonistic relation, for these reasons, cannot, be located at $t_{1} t_{2} t_{3}$, since the relation cannot be located at any of the temporal sub-locations make up $t_{1} t_{2} t_{3}$. This sets up a fatal problem for the coherence of the temporal relation: no sub-locations of the temporal relation's entire temporal location $\left(\mathrm{t}_{1} \mathrm{t}_{2} \mathrm{t}_{3}\right)$ can have anything to do with the relation, and for that reason, the non- 
platonistic temporal relation, which is not outside of time, cannot be a temporally located entity at all, which is a contradiction.

(It appears that the argument given in 2.1.1-2.1.3 apply not only to temporally extended temporal relations, but also to the temporally unextended non-platonistic temporal relations that I will discuss next in 2.2. This is because the arguments just given deal with nothing more detailed than noncomplex connections between non-identical times, which applies to any sort of noncomplex non-platonistic temporal relation, whether temporally extended or unextended.)

\subsection{Temporally located, temporally unextended, noncomplex temporal relations}

I will next discuss the position that (somehow) a non-complex nonplatonistic interrelation of $t_{1}$ and $t_{2}$ does not involve a connection across time, extending between $t_{1}$ and $t_{2}$. Rather, the interrelation of $t_{1}$ and $t_{2}$ exists only at $t_{1}$ and $t_{2}$, and not in-between $t_{1}$ and $t_{2}$. On this scenario, an interrelation of $t_{1}$ and $t_{2}$ is in time, where $t_{1}$ and $t_{2}$ are, but the noncomplex, non-platonistic relation is temporally unextended, since on this account, the temporal relation is located where and only where $t_{1}$ and $t_{2}$ are.

One thing to note before I move into my arguments is that if $t_{1}$ and $t_{2}$ are each durations (extended) (such as in the case where $t_{1}$ and $t_{2}$ are minutes that are 10 seconds apart), but the non-platonistic relation between them is temporally unextended (temporally pointsized), it is unclear how the temporally unextended non-platonistic temporal relation can relate them, since the relation would only be able to attach to one point of each duration $t_{1}$ and $t_{2}$. The nonplatonistic temporally unextended temporal relation has no extension with which it can coincide with all of $t_{1}$, or all of $t_{2}$, in its relating of $t_{1}$ and $t_{2}$. Perhaps if $\aleph_{1}$-many unextended temporal relations were involved connecting every point of $t_{1}$ to every point of $t_{2}$ (if both $t_{1}$ and $t_{2}$ have $\aleph_{1}$-many points), this issue is solved. But philosophers typically discuss relations as if one relation relates all of duration $t_{1}$ to all of duration $t_{2}$. I see this as a serious problem for temporally unextended non-platonistic (and platonistic) relations between $t_{1}$ and $t_{2}$ if $t_{1}$ and $t_{2}$ are durations. But I however will not discuss this issue further since it is irrelevant to my arguments.

I will next move to my arguments against temporally unextended relations between $t_{1}$ and $t_{2}$. In arguing that non-platonistic temporally 
unextended temporal relations between $t_{1}$ and $t_{2}$ do not exist, (where $t_{1}$ and $t_{2}$ are any non-identical times), I will merely consider the scenario where the (alleged) temporal relation, parthood, among $t_{1}$ and $t_{2}$, where $t_{1}=$ minute (part), and $t_{2}=$ hour (whole), is a temporally unextended, noncomplex, non-platonistic temporal relation. On this account, the connection among $t_{1}$ and $t_{2}$ is a connection among nonidentical temporal locations (times that are interrelated across a temporal distance), since pieces of $t_{2}$ do not temporally overlap with $t_{1}: t_{1}$ (part) is located within $t_{2}$ 's (whole's) locations, but $t_{1}$ is not identical to many of the locations that make up $t_{2}$, such as the minute before $t_{1}$, and the minute after $t_{1}$ (if $t_{1}$ is not the first or last minute of the hour). For these reasons, the relation, parthood, between $t_{2}$ (whole) and $t_{1}$ (part), connects non-identical times, which is the very sort of temporal relation I am concerned with in this paper.

If a time $t_{1}$, for example, participates in the co-exemplification of polyadic properties (such as, the temporal relation Parthood), in such a case that instantiation of the relation in question at $t_{1}$ is only at $t_{1}$. If one of the temporally located temporally unextended relation's relata are not identical to time $t_{1}$, then $t_{1}$ is not a relatum of the relation. Similarly, if time $t_{2}$ is a temporal location, then in such a case, that instantiation of the relation in question at $t_{2}$ is only at $t_{2}$.

These restrictions imply that any non-identical times, $t_{1}$ and $t_{2}$, could not be related by a noncomplex, temporally unextended, nonplatonistic temporal relation, for the following reasons. Since $t_{1} \neq t_{2}$, and since on this account the non-platonistic interrelation of $t_{1}$ and $t_{2}$ is not being considered as temporally extended between $t_{1}$ and $t_{2}$, but only at the temporal locations $t_{1}$ and $t_{2}$, then $t_{1}$ and $t_{2}$ apparently cannot have any sort of dealings with one another (such as being interrelated by the temporal relation, parthood). It appears that in order for $\mathrm{t}_{1}$, for example, to co-exemplify a temporally unextended relation of the sort I am discussing here, which is a non-platonistic, noncomplex, non-platonistic temporal relation shared with $\mathrm{t}_{2}, \mathrm{t}_{1}$ must also be identical to $t_{2}$, and thus must apparently take on characteristics that are self-contradictory: $t_{1}$ is identical to itself and is not identical to itself). Similarly, in order for $t_{2}$ to share a temporally unextended, noncomplex, non-platonistic temporal relation with $\mathrm{t}_{1}, \mathrm{t}_{2}$ must also be identical to $\mathrm{t}_{1}$, and thus must apparently take on characteristics that involve contradiction.

If my reasoning in this sub-section is correct, it is apparently the case that noncomplex, temporally unextended, non-platonistic tempo- 
ral relation relations cannot account for any connection or relatedness among $\mathrm{t}_{1}$ and $\mathrm{t}_{2}$.

\subsection{A complex temporal relation as an extended continuum of non- complex temporal relations}

Since noncomplex temporal relations make up complex temporal relations, it may appear that non-platonistic complex relations between or among $t_{1}$ and $t_{2}$ are also impossible. But there may be varieties of temporally located complex temporal relations between $t_{1}$ and $t_{2}$ not susceptible to the problems discussed up to this point in the paper. In subsections 2.1 and 2.2 I discussed apparent serious problems with noncomplex non-platonistic temporal relations between or among $t_{1}$ and $t_{2}$, where those non-platonistic noncomplex temporal relations were considered as either temporally extended or temporally unextended. In the case of temporally extended noncomplex non-platonistic temporal relations, the apparent problems I discussed drew from the combination of the partlessness and temporal extendedness (extended larger than one basic building block of time) of non-complex temporally located temporal relations. In the case of temporally unextended noncomplex, non-platonistic relations, the apparent problems I discussed drew from noncomplex temporal relations not being able to connect $t_{1}$ and $t_{2}$ if non-platonistic, temporal noncomplex temporal relations are not in any way temporally extended between relata. Perhaps a complex non-platonistic temporal relation of a very specific sort can avoid these problems.

The following two sorts of temporally located, temporally extended, complex temporal relations between or among $t_{1}$ and $t_{2}$ may avoid the problems of noncomplex non-platonistic temporal relations I discussed in subsections 2.1 and 2.2.

1. A non-platonistic relation composed of an extended continuum of durationless (point-sized), non-complex, non-platonistic temporal sub-relations between $t_{1}$ and $t_{2}$. (Any one of these non-platonistic durationless sub-relations are temporal since they are in time (they are non-platonistic), but they are durationless in that the location in time that any one of them occupies is temporally unextended.)

2. A non-platonistic relation composed of discrete temporal subrelations in tandem between $t_{1}$ and $t_{2}$, where the sub-relations have a basic (irreducible) duration (a basic temporal size, such as the size of a Planck time). 
Points 1 and 2 describe a temporal relation between $t_{1}$ and $t_{2}$ that is a succession, or chain, of temporal sub-relations in tandem, linked one after the other, by analogy as chain links are linked to give rise to a chain. (Interestingly, Loux uses 'link' to denote the tying of relations to other relations in one particularly interesting passage. (Loux 1998, 38-41)) This is not the sort of relation that I have seen discussed often in the literature, other than for a few specific cases. ${ }^{8}$ In this subsection, I will consider continuous complex temporal relations (point 1 above) (I do this in 2.3.1 and 2.3.2), and I will consider a complex temporal relation as being composed of discrete noncomplex Planckscale-sized temporal sub-relations (point 2 above) (I do this in 2.3.3). If some of the current leading theories of quantum gravity are correct (such as some of the string theories, which might be described by noncommutative geometries), there are no point-sized entities involved in the makeup of space or time, since at the Planck scale, the smallest entity is a Planck length $\left(1.6 \times 10^{-35} \mathrm{~m}\right)$ or Planck time $\left(10^{-43}\right.$ s). ${ }^{9}$ I will only consider the noncomplex sub-relations to be Planck size or smaller, since if the noncomplex sub-relations were larger than that, they would occupy more than one location of time, and the problems of subsections 2.1 and 2.2 would ensue. Physicists and philosophers take each position seriously: the position that (1) time can involve durationless (temporally point-sized) items, such as time points, or perhaps durationless temporal sub-relations; and the position that (2) time only involves discrete items, and the basic building blocks of time are discrete times, and for that reason sub-relations must be discrete sub-relations of an irreducible non-zero duration $\left(10^{-43} \mathrm{~s}\right)$. Since both position are taken seriously, I will consider each scenario: the position that the noncomplex sub-relations that compose the complex relation between $t_{1}$ and $t_{2}$ are durationless (point-sized), and the position that there are noncomplex sub-relations that must be the size of a Planck time. I will find that in either case, such continuous or discrete non-platonistic noncomplex sub-relations cannot compose a complex non-platonistic relation between $t_{1}$ and $t_{2}$.

\footnotetext{
${ }^{8}$ Some accounts of causation are described as this sort of a relation.

${ }^{9}$ To my knowledge, even though quantum gravity theories are not verified by experimental data, many physicists are very confident that there is a Planck level. There are, however, conceptual problems with it, as Zeno showed, in the paradox of the stadium.
} 
2.3.1 A complex temporal relation as a continuum of durationless noncomplex sub-relations, part 1

I will next discuss reasons why a non-platonistic complex temporal relation (allegedly) connecting $t_{1}$ and $t_{2}$ that is composed of $\aleph_{1}$-many durationless noncomplex sub-relations apparently cannot constitute a temporal relation between $t_{1}$ and $t_{2}$.

It might seem that $\aleph_{1}$-many noncomplex sub-relations constituting a temporally located complex relation between $t_{1}$ and $t_{2}$ would be a complex relation that consists of durationless sub-relations that directly link to one another, in order to give rise to a temporally extended relation between $t_{1}$ and $t_{2}$. But if that were the case, the temporally located complex relation would be denoted by a statement that describes an infinite regress of durationless sub-relations: ' $t_{1}$ is related to a sub-relation that is related to another sub-relation that is related to another sub-relation...' This may, however, imply that $\mathrm{t}_{1}$ and $t_{2}$ are not related, since there is no last step in this regress of durationless sub-relations between $t_{1}$ and $t_{2}$, and thus $t_{1}$ and $t_{2}$ would be unrelated. This infinite regress attempts to complete a task by an infinite sequence of steps, where the 'completion' 'at infinity,' some might claim, in fact never occurs, since an infinite set of items has no last item. Chisholm considers this sort of regress vicious; Moreland has lucidly written about Chisholm's position:

There are at least three forms of infinite regress arguments... [One form] involves claiming that a thesis generates a 'vicious' infinite regress. How should 'vicious' be characterized here?... Roderick Chisholm says that 'One is confronted with a vicious infinite regress when one attempts a task of the following sort: Every step needed to begin the task requires a preliminary step.' [Chisholm, 1996, p. 53.] For example, if the only way to tie together any two things whatever is to connect them with a rope, then one would have to use two ropes to tie the two the two things to the initial connecting ropes, and use additional ropes to tie them to these subsequent ropes, and so on. According to Chisholm, this is a vicious infinite regress because the task cannot be accomplished. (Moreland 2001, 24.) (Emphasis added.)

\subsubsection{A complex temporal relation as a continuum of durationless noncomplex} sub-relations, part 2

Some philosophers consider infinities to involve paradoxes, and for that reason, they make a point to avoid infinities when describing 
collections. But others may object to such a position and to the reasoning given in 2.3.1, and may hold that infinite collections can exist in nature. Examples of such collections might be, for example, the collection of spatial locations, the collection of time-instants before this present moment, ${ }^{10}$ or, perhaps, the collection of noncomplex durationless sub-relations constituting a temporally extended complex temporal relation between or among $t_{1}$ and $t_{2}$.

An extended continuum of durationless temporal sub-relations resembles an extended continuum of topological spatial points. Such a complex temporal relation consists of $\aleph_{1}$-many temporally unextended, temporally located, temporally non-collocated sub-relations, that give rise to an extended continuum (the complex relation between $t_{1}$ and $t_{2}$ ). For these reasons, hereafter I will consider a complex relation that is composed of $\aleph_{1}$-many durationless temporally noncollocated sub-relations to be a complex temporal relation that is a continuum of durationless sub-relations. Points in a continuum do not directly contact one another, since any point in a continuum is not immediately next to any other points. This reasoning would apply to an extended continuum of temporally located durationless temporally non-collocated sub-relations extending between $t_{1}$ and $t_{2}$ : none of the $\aleph_{1}$-many durationless temporally non-collocated sub-relations are immediately next to one another. For this reason, a complex relation composed only of durationless temporally non-collocated subrelations cannot give rise to a complex connection between $t_{1}$ or $t_{2}$.

Continuums of points are, however, typically considered to be composed of interrelated points. ${ }^{11}$ Perhaps, as with the point-set topological account of space, the complex relation between $t_{1}$ and $t_{2}$ could consist of $\aleph_{1}$-many interrelated temporally non-collocated point-sized sub-relations. If so, perhaps the reasoning of the previous paragraph, where $\aleph_{1}$-many temporally non-collocated sub-relations were considered to be the only constituents of a continuum is misguided. ${ }^{12}$ Instead of discussing the durationless temporally non-collocated sub-

${ }^{10}$ This is a position discussed extensively by Quentin Smith $(1995,1993)$.

${ }^{11}$ Grünbaum (1952, 2001a, 2002b) is one of the philosophers who has argued for this commonly held position.

${ }^{12}$ This is typically held to be the error that Zeno made in his Measure Paradox (unextended points somehow compose an extended line, plane, or volume). See Pyle (1995, 1-7). 
relations as directly attached to one another (which is impossible), the durationless temporally non-collocated sub-relations instead should be considered as interconnected by a relation, topological connectedness, which is perhaps analogous to point-set topological accounts of connectedness of spatial points in the spatial manifold.

If a continuum is extended and interconnected, since the durationless temporally non-collocated sub-relations of the continuum cannot account for the interconnectivity of the continuum, there are two constituents of the complex temporal relation between $t_{1}$ and $t_{2}$ : (1) the $\aleph_{1}$-many durationless temporally non-collocated subrelations, and (2) the topological relation, interconnectedness, between or among the $\aleph_{1}$-many durationless temporally non-collocated subrelations. I will next argue that a non-platonistic interconnectedness relation between or among the durationless temporally noncollocated sub-relations that compose the non-platonistic complex temporal relation between $t_{1}$ and $t_{2}$ cannot connect the $\aleph_{1}$-many durationless temporally non-collocated sub-relations.

Since none of the non-platonistic durationless temporally noncollocated sub-relations are immediately next to one another, the interconnectedness relation between or among the durationless temporally non-collocated sub-relations is a relation between or among nonidentical sub-relations (the sub-relations are at a temporal distance from one another). If connectedness is a relation between or among the temporally non-collocated sub-relations, and if the connectedness relation is not also a complex non-platonistic temporally extended relation composed of a $\aleph_{1}$-many durationless sub-relations, in order to interconnect the durationless sub-relations, the connectedness relation would be a non-platonistic noncomplex relation between non-collocated subrelations, which is for that reason located at more than one temporal location. But this is exactly the sort of relation found to apparently involve contradiction in subsections 2.1 and 2.2.

For these reasons, the relation, connectedness, connecting the $\aleph_{1^{-}}$ many durationless temporally non-collocated sub-relations must also be a complex relation consisting of $\aleph_{1}$-many durationless temporally non-collocated sub-relations that are not directly linked to one another. If the connectedness between or among the durationless temporally non-collocated sub-relations was also composed of durationless sub-relations, the relation, connectedness, would itself provide no continuous connection between the non-collocated durationless temporally non-collocated sub-relations that compose the complex 
relation between or among $t_{1}$ and $t_{2}$. Only if the durationless temporally non-collocated sub-relations that compose connectedness were also interconnected by a complex relation, connectedness ${ }_{2}$ (where connectedness $s_{2}$ is also composed of continuum-many durationless temporally non-collocated sub-relations), would connectedness provide a continuous connection of the durationless sub-relations between or among the complex temporal relation connecting $\mathrm{t}_{1}$ and $\mathrm{t}_{2}$. Connectedness ${ }_{2}$ would need connectedness $s_{3}$, and an infinite regress would ensue, where each connectedness relation would require another instantiation of connectedness. At any stage of the regress, each instantiation of the connectedness relation is composed of $\aleph_{1}$-many durationless temporally non-collocated sub-relations that do not directly link to one another, which require another instantiation of the connectedness relation. The problem, however, is that any stage of the regress only consists of unconnected $\aleph_{1}$-many durationless temporally non-collocated subrelations, none of which are in contact. At any stage, the unconnected sub-relations require another distinct relation at the next stage of the regress to hold it together, but where the relation at the next stage is also composed of $\aleph_{1}$-many unconnected durationless sub-relations that are not in contact. Every stage of the regress is only composed of unconnected $\aleph_{1}$-many durationless (point-sized) elements (subrelations), and for that reason, nowhere in the regress is there any contact or connection between any sub-relations, and there is no interrelating at all between $t_{1}$ and $t_{2}$. In other words, since we never arrive at a stage in the regress where there are anything but $\aleph_{1}$-many durationless sub-relations that are not linked to one another, the temporal connectedness among the $\aleph_{1}$-many sub-relations that compose the complex relation connecting $t_{1}$ and $t_{2}$ is apparently impossible. I do not know of any other way to consider a continuous relation between $t_{1}$ and $t_{2}$, and for that reason I will move to the other case: a complex relation between $t_{1}$ and $t_{2}$ composed of discrete temporal sub-relations in tandem.

\subsubsection{A complex temporal relation composed of planck-sized sub-relations}

In this subsection I consider the complex relation between $t_{1}$ and $t_{2}$ to be a succession of discrete sub-relations that are the size of the discrete basic building blocks of time that Planck-scale physicists discuss. I will argue that there are no complex, non-platonistic temporal relations between $t_{1}$ and $t_{2}$, if the complex temporal relation is composed of a tandem of discrete noncomplex sub-relations. To see why this is the 
case, I only need to consider the minimum case, where two directly adjacent Planck times, call them $t_{1}$ and $t_{2}$, are interconnected, which I will do next.

According to quantum gravity theories, ${ }^{13}$ since there is no time smaller than a Planck time, there is no time between any two Planck times that are directly adjacent. The smallest sub-relation that can be considered to connect two adjacent Planck times would not be smaller than a Planck time and thus would be a sub-relation that is itself an irreducible (noncomplex) non-platonistic relation between the two directly adjacent Planck times, $t_{1}$ and $t_{2}$. This one Planck-sized sub-relation would coincide with both of $t_{1}$ and $t_{2}$ (see Figure 1).

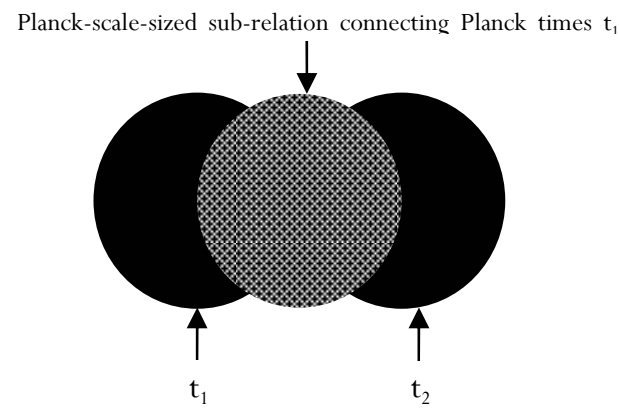

Figure 1

It cannot be the case that one irreducible (noncomplex) subrelation coincides with more than one Planck time, since in connecting $t_{1}$ and $t_{2}$, the sub-relation is (i) noncomplex, and (ii) must coincide with $t_{1}$ and $t_{2}$ in order to connect them (see Figure 1). If the relation is located at $t_{1}$ and $t_{2}$, which it appears it must be if it is to connect to them, then this relation is a noncomplex relation connecting two nonidentical Planck times, which is exactly the sort of relation I found to be contradictory in the previous subsections of this section, and for that reason I will not discuss it further.

The only way this problem could be avoided is if the Planck sized sub-relations exactly coincided with Planck-sized basic building blocks

\footnotetext{
${ }^{13}$ Quantum gravity is a unification of quantum theory and relativity, and is for that reason believed to be the theory that will end the divergence that exists in physics between relativity and quantum mechanics. See Lesniewski (1997), Kane (2000), Madore (2000), and Jones and Moscovici (1999).
} 
of time, linking up to one another while they each exactly occupy only one discrete basic building block of time. But on this account, it is unclear how these discrete sub-relations could link to one another. They cannot link, in this case, by partial collocation, since they only exactly coincide with non-identical Planck times. Without coinciding, the Planck-sized sub-relations are entirely non-collocated, and for that reason, it is unclear how the Planck-sized sub-relations can be relata of one another without linking (to use Loux's word) in any way to one another. If we imagine that the sub-relations link by abutting to one another without overlapping, there must be an item distinct from the sub-relations that is responsible for holding (or 'gluing') the subrelations together if the sub-relations perfectly coincide with Planck times $t_{1}$ and $t_{2}$ and abut without overlapping. However, this item responsible for holding the Planck-sized sub-relations together would be smaller than a Planck time, and in fact would be durationless if the sub-relations abut, and for that reason this gluey item would be susceptible to the problems to do with durationless sub-relations given above in this sub-section. For example, if the gluey item is durationless (point-sized), it is unclear how it could contact and link the two sub-relations that exactly coincide with Planck times $t_{1}$ and $t_{2}$, since durationless (point-sized) items cannot contact any other entities unless the entities that contact the point-sized gluey item collocate with the gluey item, which would mean that the sub-relations partially collocate (temporally overlap).

If we go against quantum gravity theorists and imagine that the discrete temporal sub-relations of the complex temporal relation between $t_{1}$ and $t_{2}$ are somehow larger than a point, but smaller than the basic discrete times of the Planck scale, the same problems as those just described involving Planck-scale-sized sub-relations would ensue. In other words, regardless of the size of the discrete basic building blocks of time or the sub-relations connecting the discrete basic building blocks of time, discrete basic building blocks of time that are interconnected by discrete sub-relations would involve the problems to do with Planck-sized sub-relations and Planck times just discussed.)

If my reasoning in this section is correct, there apparently cannot be any temporal relations between $t_{1}$ and $t_{2}$ if the relations are nonplatonistic relations. 


\subsection{Non-platonistic monadic relatedness}

Some philosophers may argue that according to an account of nonplatonistic monadic relatedness, non-platonistic monadic properties located at $\mathrm{t}_{1}$ or $\mathrm{t}_{2}$, rather than non-platonistic relations at both $\mathrm{t}_{1}$ and $\mathrm{t}_{2}$ account for a relatedness of times, and for that reason, non-platonistic monadic relatedness may be assumed to avoid the problems discussed so far in this section to do with relations between times. I will next argue that an account of non-platonistic monadic relatedness apparently involves serious problems.

An example of a non-platonistic temporal monadic property might be, at a temporal distance from $t_{2}$, possessed by, for example, $\mathrm{t}_{1}$. If the monadic property is instantiated by a time, such as time $t_{1}$, then according to an account of non-platonistic monadic relatedness, the non-platonistic monadic property, at a temporal distance from $t_{2}$, is not at $t_{2}$, and is at $t_{1}$ (and at any other time that is at a distance from $t_{2}$ ). The problem that I will discuss to do with non-platonistic monadic relatedness has to do with the issue that a monadic property, such as the property, at a temporal distance from $t_{2}$, is not located at $t_{2}$ but has involvement with both $\mathrm{t}_{1}$ and $\mathrm{t}_{2}$ : the monadic property has involvement with $t_{1}$ because it is instantiated by $t_{1}$ and therefore located at $t_{1}$; and the monadic property has involvement with $t_{2}$ because $t_{2}$ 's existence (allegedly) makes $t_{1}$ a certain way ${ }^{14}$ : at a temporal distance from $t_{2} \cdot t_{2}$ 's making $t_{1}$ a certain way can be considered a sort of involvement that $t_{2}$ has with the non-platonistic monadic property, even though the nonplatonistic monadic property is not located at $t_{2}$ for the following reasons. Time $t_{2}$ must exist if it is to be the referent of ' $t t_{2}$ ' in the statement that describes the monadic property, 'at a temporal distance from $t_{2}$. For that reason, some sort of connection between time $t_{2}$ and the monadic property must exist in order for $t_{2}$ to be referred to in the statement that describes the monadic property, 'at a temporal distance from $t_{2}$.' If the monadic property is at $t_{1}$, but the subject of

\footnotetext{
${ }^{14}$ This is in fact the definition of a property: a property makes a particular a certain way. Armstrong discusses how properties are ways objects (substances) are:

Properties are ways things are. The mass or charge of an electron is a way the electron is... Relations are ways things stand to each other.

If a property is a way that a thing is, then this brings the property into very intimate connection with the thing, but without destroying the distinction between them. (Armstrong, 1989, 96-97.) (Emphasis added.)
} 
the monadic property is $t_{2}$, then the relation just mentioned in the previous sentence is a relation that is between or among non-identical times, which is the sort of relation I argued above does not exist.

\section{Platonistic relations between non-identical times}

\subsection{Platonistic temporal relations between or among non-identical times}

To avoid the problems discussed in section 2, temporal relations among $t_{1}$ and $t_{2}$ could be considered temporal relations that are not in time. Rather, relations among $t_{1}$ and $t_{2}$ could be considered to be temporally unlocated universals (platonic universals) exemplified by $t_{1}$ and $\mathrm{t}_{2}$, and not at $\mathrm{t}_{1}$ or $\mathrm{t}_{2}$. On this account, $\mathrm{t}_{1}$ and $\mathrm{t}_{2}$ are (allegedly) interrelated since they co-exemplify a platonistic relational property. This sort of relation between $t_{1}$ and $t_{2}$ is, in the platonic sense, in the temporally unlocated platonic realm. Considering platonistic relations as temporally unlocated is the standard position on platonia. In using the word 'non-temporal' to mean 'not in time,' Grossmann, a major platonist philosopher, writes:

According to Plato, as we have seen, there are two realms: the realm of temporal things, of things which exist in time, and the realm of atemporal things, of things which do not exist in time. To the first realm belong the individual things around us; to the second, their properties [including their polyadic, or relational, properties]. (Grossmann 1990, 5)

Plato... speaks of 'abstract quality'. I shall speak of abstract things (entities, existents) in general. An abstract thing is a thing which is neither temporal nor spatial. A concrete thing, on the other hand, is a thing which is temporal and/or spatial. (Grossmann 1990, 7)

...[P]roperties... are abstract things; they are not spatio-temporal. It follows that they do not belong to the universe. They are not part of the universe. (Grossmann 1990, 8$)^{15}$

In this section, I will argue that a platonistic account of temporal relations between or among $t_{1}$ and $t_{2}$ is a contradictory account of

\footnotetext{
${ }^{15}$ Moreland (2001), also a platonist, discusses Grossmann's platonism in depth, especially on pages $4,9,12-13,102-103$, and many other places.
} 
temporal relations. I will not argue against the existence of temporally unlocated objects. Rather, I will only argue that any sort of (alleged) connection between times that is a platonistic connection involves serious problems.

The co-exemplification of atemporal relations by $t_{1}$ and $t_{2}$, on the platonistic account, involves the platonistic exemplification tie, which is a tie connecting universals in the temporally unlocated platonistic realm (where relations such as 10 seconds apart, or parthood, and any other platonistic relation, are) to entities in the temporal realm, such as times $t_{1}$ and $t_{2}$. In this case, where $t_{1}$ and $t_{2}$ co-exemplify a temporal relation, platonistic exemplification is a tie between or among temporally unlocated universals (platonic universals) and times (which are platonistic thin particulars $\left.{ }^{16}\right)$. In this section, I will argue that there is a spe-

${ }^{16}$ Platonists often neglect to reveal what exactly a first-order property ties to, and platonists often merely claim that it is 'the particular that exemplifies properties.' (This also applies to relational properties, such as those I am discussing in this article. First order properties, including first-order relational properties, are properties that are not properties of other properties.) But this is not precise, for the following reasons. First-order platonic properties cannot be tied to other properties, lest a platonistic substance (such as times $\mathrm{t}_{1}$ and $\mathrm{t}_{2}$ ) be a wholly temporally unlocated bundle of compresent properties (on the bundle account of objects, first-order properties are not tied to a thin particular, but rather are tied to one another). Thus, first-order properties must tie to the only remaining element of the substance: the particularity. Since this particularity cannot be a property (lest a substance be a bundle), this particularity can only be the thin particularity of the substance. Accordingly, a time is a temporal entity in the sense that it is a thin particular (wholly temporal) exemplifying platonic universals (wholly temporally unlocated). In this way, platonistic metaphysics only involves wholly temporal items or wholly atemporal items, and in considering a time as wholly temporal, I am referring to the thin particular that is wholly temporal, and which is distinct from, but tied to, wholly temporally unlocated properties.

A thin particular is typically discussed in the context of non-platonistic metaphysics of objects, such as the Aristotelian tradition (Armstrong 1989, 60) and Armstrongian physicalism, but I will discuss it as the item in platonistic metaphysics that is the literal exemplifier properties. I see no objection in using it here in the context of platonism with one minor modification: the properties exemplified are platonistic, not Aristotelian. Other philosophers use Armstrongian concepts in a platonistic context. For example, Vallicella (2000), a platonist, discusses Armstongian ontology extensively, accordingly intermixing the two due to Vallicella's platonism, including using the concept of a thin particular. Moreland discusses thin particulars:

[Armstrong] distinguish[es] a thick from a thin particular. A thick particular is a state of affairs (e.g., A's being F), and as such it is a particular along with its 
cific problem to do with the platonistic account of polyadic property possession since, I will discuss, there may be a fatal problem involved with the exemplification tie that ties times and temporally located platonistic properties. ${ }^{17}$ If I am correct, and if the problem is serious enough, a temporally unlocated platonistic relation cannot relate $t_{1}$ and $\mathrm{t}_{2}$.

\subsection{Relations and unmediated attachments}

In this sub-section I discuss how I use the terms 'exemplification tie' and 'unmediated attachment,' which are terms relevant to the discussion of any (alleged) platonistic interrelation of non-identical times $\left(t_{1}\right.$ and $\mathrm{t}_{2}$ ).

There are two entities (in the broadest sense of 'entity') that I will be concerned with in discussing the exemplification tie between temporally unlocated platonic universals and times $t_{1}$ and $t_{2}$.

i) The exemplification tie, which is an intermediary that ties a time (which is a platonistic thin particular), and the temporally unlocated

properties. The particular 'enfolds' its properties in the sense that they are spatially located where the thick particular is. In the statement 'this is hot.' the word 'this' refers to a thick particular and says that hotness is among its properties. The thin particular is the particular considered in abstraction from all its properties. It is not a thing per se, but amounts to bare numerical difference or thisness, the individuating factor that makes the thick particular more than just a bundle of universals. (Moreland 2001, 87)

A 'platonistic thin particular' would be different from an Armstrongian thin particular in that, unlike the Armstrongian thin particular, platonistic universals, if they exist, are not required to be part of a thick particular since platonic universals can (allegedly) be unexemplified. On Armstrong's account of a thin and thick particular, '[u]niversality and particularity are, he says, inseparable aspects of all existence, they are neither reducible nor related to each other and, although distinct, their union is closer than a relation.' (Moreland 2001, 86) I do not use 'thin particular' in a platonistic metaphysics to confuse Aristotelian and platonistic states of affairs, but rather to be clear in what I mean: the platonistic scenario is: a spatially located entity (a platonistic thin particular) is tied (exemplification) to spatially unlocated entities (platonic universals). Also, I use 'thin particular' here in the context of platonism because I find that platonists very rarely discuss the analogue of the thin particular in platonistic metaphysics.

${ }^{17}$ Hereafter, I refer to exemplification as a tie, and not a relation, for reasons given in a citation below from Loux. 
platonistic n-adic temporal properties (monadic properties such as, relatedness, or relations, such as, 10 seconds apart, or parthood).

ii) Unmediated attachments, which temporally located platonistic thin particulars and the exemplification tie are involved in, and which a temporally unlocated platonic universal and the exemplification tie are involved in (or which, as I will explain below, parts of the platonistic exemplification tie, if it has parts, might be involved in). Unmediated attachment describes the attachment between the exemplification tie and the platonistic tin particular, and the exemplification tie and the platonistic universal.

Let 'unmediated attachment' express the concept of an attachment which times and the exemplification tie are involved in, and which platonistic universals and the exemplification tie are involved in, and which does not involve an intermediary. An unmediated attachment is not a relation between the exemplification and the times, or between the exemplification tie and the universal, and unmediated attachment does not involve non-relational ties, or any sort of item that is between the exemplification and the times, or between the exemplification tie and the universal. Unmediated attachment is normally how exemplification is conceived to attach to a property or to platonistic (thin) particulars. The concept of unmediated attachment comes from responses to F.H. Bradley's work on the paradox of the relations regress. Loux lucidly explains:

According to the [platonist], for a particular, $a$, to be $F$, it is required that both the particular, $a$, and the universal, $F$-ness, exist. But more is required; it is required, in addition, that a exemplify $F$-ness. As we have formulated the [platonist's] theory, however, $a$ 's exemplifying $F$-ness is a relational fact. It is a matter of $a$ and $F$-ness entering into the relation of exemplification. But the realist insists that relations are themselves universals and that a pair of objects can bear a relation to each other only if they exemplify it by entering into it. The consequence, then, is that if we are to have the result that $a$ is $F$, we need a new, higher-level form of exemplification (call it exermplification ${ }_{2}$ ) whose function it is to insure that $a$ and $F$-ness enter into the exemplification relation. Unfortunately, exemplification ${ }_{2}$ is itself a further relation, so that we need a still higherlevel form of exemplification (exemplification ${ }_{3}$ ) whose role it is to insure that $a, F$-ness, and exemplification are related by exemplifiaction ${ }_{2}$; and obviously there will be no end to the ascending levels of exemplification that are required here. So it appears... that the only way we will ever 
secure the desired result that $a$ is $F$ is by denying that exemplification is a notion to which the realist's theory applies.

The argument just set out is a version of the famous argument developed by F.H. Bradley. Bradley's argument sought to show that there can be no such things as relations... [Platonists] claim that while relations can bind objects together only by the mediating link of exemplification, exemplification links objects into relational facts without the mediation of any further links. It is, we are told, an unmediated linker; and this fact is taken to be a primitive categorial feature of the concept of exemplification. So, whereas we have so far spoken of exemplification as a relation tying particulars to universals and universals to each other, we more accurately reflect the realist thinking about the notion if we follow realists and speak of exemplification as a 'tie' or 'nexus' where the use of these terms has the force of binging out the nonrelational nature of the linkage this notion provides. (Loux 1998, 38-41.) ${ }^{18}$

Exemplification is a non-relational tie or nexus ${ }^{19}$ between or among properties and platonistic thin particulars, or between or among properties and other properties. In this case, where $t_{1}$ and $t_{2} c-$ exemplify a temporal relation, the exemplification tie is not related to the relation (connectivity, 10 seconds apart, parthood) or to the nonidentical times $\left(t_{1}\right.$ and $\left.t_{2}\right)$. And the exemplification tie is not a relation between or among the platonistic temporal relation (connectivity, 10 seconds apart, parthood) and the non-identical times $\left(\mathrm{t}_{1}\right.$ and $\left.\mathrm{t}_{2}\right)$. Given the exemplification tie's apparent non-relational nature, in this paper, I will discuss exemplification as a tie, rather than as a relation.

To avoid a Bradley-esque regress in the scenario where $t_{1}$ and $t_{2}$ are interrelated platonistically, four entities are involved: (a) $t_{1}$, (b) $t_{2}$, (c) the relation (connectivity, parthood), (d) the exemplification tie which involves an unmediated attachment to both $t_{1}$ and $t_{2}$, and which involves an unmediated attachment to the relation. Exemplification is a tie, and apparently is not a relation, because the exemplification tie

${ }^{18}$ I have altered Loux's passage to read as if he only discusses platonic realism, rather than metaphysical realism in general. For further lucid discussion on these issues, see Vallicella (2000). Some argue that it is not so certain that Bradley did not in fact conclusively argue that relations do not exist, and they doubt that exemplification does away with the problems Bradley disclosed. See Grupp (2003).

${ }^{19}$ Moreland (2001, 99-100) also refers to exemplification as a 'nexus,' but unlike Loux, he typically refers to it as a relation. 
allegedly holds the relation and non-collocated temporal entities together without the Bradley-esque regress ensuing.

The relation (10 seconds apart, parthood) does not involve an unmediated attachment to the times. Rather, the relation (10 seconds apart or parthood) involves unmediated attachments to the exemplification tie. Likewise, the interrelated entities $\left(t_{1}\right.$ and $\left.t_{2}\right)$ are not involved in unmediated attachments to the relation (10 seconds apart, parthood). Rather, the interrelated entities $\left(t_{1}\right.$ and $\left.t_{2}\right)$, and the relation (10 seconds apart, parthood), involve an unmediated attachment to the exemplification tie. The relation (10 seconds apart, parthood), and interrelated entities $\left(t_{1}\right.$ and $\left.t_{2}\right)$, do not involve unmediated attachments to each other; rather these together form an unordered set [relation (10 seconds apart or parthood), object $\mathrm{t}_{1}$, object $\left.\mathrm{t}_{2}\right]$. The members of this set involve unmediated attachments to the exemplification tie, in such a way as to constitute the interrelated entities $\left(t_{1}\right.$ and $\left.t_{2}\right)$ being interrelated with each other. Here 'being' and 'with,' in '....being interrelated with..., denote the exemplification tie.

It is worth emphasizing these distinctions for the sake of further clarifying what is meant by 'exemplification.' We refer to the exemplification tie when we say that the interrelated entities $\left(t_{1}\right.$ and $\left.t_{2}\right)$ are interrelated (...are...). The exemplification tie is also expressed when we say that the interrelated entities stand in a relation to each other; we use 'stands in... to' to denote the exemplification tie that involves unmediated attachments with the spatially unlocated relation, and with the platonistic thin particulars. 'Two things $t_{1}$ and $t_{2}$ stand in the relation R' means 'the two things exemplify the relation R.' The ontological role of the exemplification tie is to act as the nonrelational intermediary between $(A)$ the interrelated entities $\left(t_{1}\right.$ and $\mathrm{t}_{2}$ ), and (B) the relation (10 seconds apart, parthood) without a Bradleyeqsue regress ensuing. (To my knowledge, platonists have not told us how the exemplification tie ties without being related to property and particular, but have merely asserted that: in order for platonism to be coherent, the exemplification tie must somehow tie non-relationally. ${ }^{20}$ )

\footnotetext{
${ }^{20}$ Some readers may be concerned that any description of the exemplification tie is not possible since the tie is alleged to be primitive. I suggest that if this is the case, then an inquiry of the nature of the exemplification tie will reveal its primitivism. As an aside, I however maintain that the primitivism of the exemplification tie has not been established, perhaps due to the near absence of discussion of the tie. Rather, it appears that it has been merely asserted that the exemplification tie is primitive,
} 
Some may object that the reasoning given in the passage above by Loux is fatally flawed, since 'unmediated attachment' must have a truthmaker, but if there is a verbal referent to 'unmediated attachment,' then an unmediated attachment, as described by Loux and myself above, is impossible, since unmediated attachment would refer to yet another entity (in the broadest sense of 'entity'), distinct from the universal, the exemplification tie, and the particulars. This objection fails, however, for the following reasons. The referent of 'unmediated attachment,' if I understand Loux's terminology correctly, is not another entity distinct from the exemplification tie, property, and particular, but is a manner or way in which the property and the exemplification tie, or particular and the exemplification tie, are linked, to use Loux's word. For example, in his passage above, Loux describes the exemplification tie as a 'linker,' and the word 'link' might imply a chain-like connection, to use a rough analogy, where only the pieces of a chain are involved, and a third mediating entity, between chain links, such as a glue or string, holding chain links together and which is an entity different from the chain links, is not required for the linking of the chain links to ensue.

Lastly, the exemplification tie is not merely a non-relational unmediated attachment of a property with platonistic thin particulars. When we say, ' $t_{1}$ and $t_{2}$ share $R$,' there must be a truthmaker denoted by 'share.' For this (and other) reasons, the exemplification tie is an additional entity (in the broadest sense of the word 'entity'), in addition to the atemporal property and the platonistic thin particulars, which connects the platonistic factor of thin particularity to the platonistic temporally unlocated universal. Some may object here, and maintain that it is correct to discuss this scenario as if relations directly attach to one another, or to particulars, rather than as if relations and their relata are mediated by an exemplification tie. This would be to consider 'the unmediated attachment of a relation to its relata' as synonymous with 'exemplification tie,' where an unmediated attachment between a relation and its relata is an entity (in the broadest sense of 'entity') that is a special 'unmediated linkage' that a relation and its relata are involved in. However, to my knowledge, this cannot be how the exemplification tie is to be considered, for if it were, the atemporal relation would be involved in an unmediated attachment with 
times $\left(t_{1}\right.$ or $\left.t_{2}\right)$, and in order to directly attach to time $\left(t_{1}\right.$ or $\left.t_{2}\right)$, the atemporal relation would have to be at the location of the where a time $\left(t_{1}\right.$ or $\left.t_{2}\right)$ is located, if it is to have an unmediated attachment with the time. By this I mean that the time is only where it is, and is of course only found in the time series, and if something is to have an unmediated attachment with it, that something can only do so if it is where the time is. If it is not where the time is, it cannot have an unmediated attachment with the time. Rather, only items which are right where a time is can have an unmediated attachment to the time. But if this is the case, the atemporal relation, in going to where the time is, would be located in time, and would be an atemporal item that is located in time, which is impossible.

\subsection{Platonistic exemplification ties and unmediated attachments}

In this section, I will further discuss the following unmediated attachments:

(i) The unmediated attachment between the exemplification tie and platonistic thin particulars,

(ii) The unmediated attachment between the exemplification tie and platonistic temporal relation, and

(iii) The unmediated attachment between the parts of the exemplification tie (if the exemplification tie has parts).

In this subsection, I will discuss that one of these unmediated attachments involved in platonistic property possession apparently involves an unmediated attachment between a wholly atemporal item and a wholly temporal item. I will also discuss that such unmediated attachments as these between wholly temporal items and wholly atemporal items are apparently impossible, and for that reason, $t_{1}$ and $t_{2}$ cannot be platonistically interrelated. One of the unmediated attachments involved in platonistic property possession is an unmediated attachment between a wholly temporal entity and a wholly temporally unlocated entity for the following reasons.

1. If the exemplification tie is partless (simple), and is either wholly temporally located or wholly temporally unlocated, ${ }^{21}$ then the ex-

\footnotetext{
${ }^{21}$ A simple (partless) platonistic exemplification is wholly temporally located, or wholly temporally unlocated, for the very reason that it is the platonistic exemplifica-
} 
emplification tie is an intermediary that connects wholly temporal entities (times $t_{1}$ and $t_{2}$ ) and the wholly temporally unlocated temporal relation, and the exemplification tie involves an unmediated attachment to both $t_{1}$ and $t_{2}$ (which are wholly temporal entities) and to the wholly temporally unlocated temporal relation (10 seconds apart, parthood)..$^{22}$ On this account, where the exemplification tie is simple, for there to be any tying of a platonistic thin particular and a temporal property, there is an unmediated attachment between a wholly temporal entity and a wholly atemporal entity.

2. If the exemplification tie is both temporally located and temporally unlocated, it is composed of two or more parts, where at least one part is wholly temporally located (and involves unmediated attachments with the $t_{1}$ and $t_{2}$ ), and where at least one part is wholly temporally unlocated (and involves an unmediated attachment with a platonic universal, such as 10 seconds apart or parthood). In order that the exemplification tie give rise to a tie between wholly temporal items (times $t_{1}$ and $t_{2}$ ) and wholly temporally unlocated platonistic universals, wholly temporal and wholly temporally unlocated parts of the exemplification tie must involve an unmediated attachment. ${ }^{23}$

tion tie, and not, for example, a time that exemplifies temporally unlocated properties, or a temporal property that is exemplified by a time. A time, according to some platonists, might be considered not to be wholly temporal, but rather to be an entity that is temporally located and temporally unlocated, since it has temporal and atemporal aspects or constituents: wholly temporally unlocated platonic universals, that are tied to (exemplified by) a platonistic thin particular (which is wholly temporal). Exemplification is not a temporal item, since it is the special tie that gives rise to time are times because they are: platonistic thin particulars are exemplify certain temporal properties. Unlike a time, that might be considered by platonists to have temporally unlocated constituents, the exemplification tie, in being a constituent of, or aspect of, those times, is wholly temporally located or wholly temporally unlocated. These same points would apply to a non-simple exemplification tie, where parts of the tie would be wholly temporally located or wholly temporally unlocated.

${ }^{22}$ Moreland, a pure realist, appears to hold this position: 'For traditional realists, neither the universal nor the exemplification nexus are spatiotemporal... [T] he exemplification nexus connects an abstract entity with a spatiotemporal one.' (Moreland 2001, 100) On this account, a wholly spatially located entity (the platonistic thin particular) and a wholly spatially unlocated entity (the exemplification tie) would involve an unmediated attachment.

${ }^{23}$ Wolterstorff (1970, Chapter 4) appears to hold that exemplification is composed of parts. 
In order for their to be a tie between a property and particular, the exemplification tie between property and particular must be unbroken, and for that reason, points 1 and 2 above both suggest that platonistic property possession must involve an unmediated attachment of a wholly temporal entity and a wholly temporally unlocated entity. It is this unmediated attachment that I will be concerned with in this subsection, and which I will find apparently contradictory.

I will not discuss which entities might be those that are specifically involved in the unmediated attachment of an entity that is wholly temporal and an entity that is wholly atemporal. I will only focus on the issue that there is at least one such unmediated attachment required in platonistic property possession, as described in points 1 or 2. I will call the entity that is wholly outside of time that is involved in this unmediated attachment, $\mathrm{O}$, and the wholly temporal entity that is involved in the unmediated attachment, $\mathrm{L}$. $\mathrm{L}$ could be the two particulars $\left(\mathrm{t}_{1}\right.$ and $t_{2}$ ), or $L$ could be the entire exemplification tie if the exemplification tie is simple and is wholly located in time, as discussed in point 1 . Or L could be a part of the exemplification tie that is in time, as discussed in point 2. O could be the platonic universal; or $\mathrm{O}$ could be the entire exemplification tie, if the exemplification tie is simple and not in time, as discussed in point 1 . Or $\mathrm{O}$ could be a part of the platonistic exemplification tie that is not in time, as discussed in point 2 above. What $\mathrm{L}$ and O symbolize depends on whether point 1 or point 2 is correct, and, beyond that, it also depends on specific details to do with points 1 and 2. In this paper, I am only concerned with the issue that on the platonistic account of property possession, there is at least one unmediated attachment between a wholly abstract entity, O, (an entity wholly outside of time), and a wholly temporal entity, L. According to my argument in the next paragraph, such an unmediated attachment, between an entity wholly in time (L) and an entity wholly outside of time $(\mathrm{O})$ is impossible (which would mean that $\mathrm{t}_{1}$ and $\mathrm{t}_{2}$ cannot be platonistically interrelated.

Since $\mathrm{L}$ is wholly located in time, $\mathrm{L}$ cannot fail to either be at a temporal location, or to be a time (in either case, $\mathrm{L}$ cannot fail to be wholly temporal). Any unmediated attachment having to do with $\mathrm{L}$ must thereby be an unmediated attachment that is wholly temporal, lest it not be a unmediated attachment to do with L. Since L can only be wholly temporal, if $\mathrm{L}$ is directly attached to any other entity, the other entity involved in an unmediated attachment with $\mathrm{L}$ cannot fail to also be in time. Since $\mathrm{O}$ is wholly outside of time, if $\mathrm{O}$ is involved in 
an unmediated attachment with $\mathrm{L}$, O must come into time and become located in time, in order to be involved in an unmediated attachment with $\mathrm{L}$. If this is the case, then $\mathrm{O}$ would be inside and not inside of time, apparently taking on characteristics that involve contradiction. A similar line of reasoning could be given when considering the unmediated attachment $\mathrm{O}$ is involved in. Unmediated attachments to do with $\mathrm{O}$ only occur by way of entities that are entirely outside of time. An entity in time, L, having any sort of dealing (such as unmediated attachment) with $\mathrm{O}$ can only do so if it is also outside of time. If this is the case, if $\mathrm{L}$ is to have an unmediated attachment with $\mathrm{O}, \mathrm{L}$ must go outside of time and become atemporal, in order to be involved in an unmediated attachment with $\mathrm{O}$. If this is the case, then $\mathrm{L}$ would be outside and not outside of time, apparently taking on characteristics that involve contradiction.

\subsection{Objection}

An objection to the argumentation in the previous subsection is treated next, and is given as follows. The platonistic property only exists in the temporally unlocated platonistic realm, and the platonistic thin particular only exists in the temporal realm, and the notion of a tie or nexus 'across' the realms, bridging the realms, is an erroneous concept. The exemplifying only exists at the times $\left(\mathrm{t}_{1}\right.$ and $\left.\mathrm{t}_{2}\right)$, and only atemporally in the platonic realm (where there relations 10 seconds apart or parthood are); and there need not be any sort of concept of bridging or literal tying from one realm to the other. For this reason, notion of an exemplification tie is misguided: the exemplification tie need not do any 'linking' or 'bridging.' For the rest of this subsection, I will use 'exemplification' in place of 'exemplification tie' in order to discuss platonistic property possession without discussing the tie from one realm to the other.

I next argue that this objection fails. The platonistic thin particular, $t_{1}$, for example, only involves an unmediated attachment to exemplification at $t_{1}$ and nowhere else, since $t_{1}$ is not identical to any other time. This unmediated attachment must be temporally located since $t_{1}$ is wholly temporal; the unmediated attachment, if not at $\mathrm{t}_{1}$, is not an attachment that can involve $t_{1}$. An unmediated attachment to the exemplification tie not at $t_{1}$ is an unmediated attachment that does not have anything to do with $t_{1}$ (whereby, exemplification would not involve an unmediated attachment with $t_{1}$ ). Since a platonistic rela- 
tion, R (parthood, 10 seconds apart), cannot fail to be temporally unlocated - call the location of the platonistic universal, $\mathrm{z}$ - this implies that $\mathrm{R}$ only involves an unmediated attachment to exemplification at $\mathrm{z}$, since $\mathrm{R}$ is nowhere else but at $\mathrm{z}$ (in the platonic realm). An unmediated attachment to exemplification not at $\mathrm{z}$ is an unmediated attachment that does not have anything to do with $\mathrm{R}$ (whereby, exemplification would not involve an unmediated attachment with $\mathrm{R}$ ).

This implies that $\mathrm{t}_{1}$ cannot exemplify $\mathrm{R}$ : if $\mathrm{R}$ only involves an unmediated attachment to exemplification at $z$, and if $t_{1}$ only involves an unmediated attachment to exemplification at $\mathrm{t}_{1}$, and if the exemplifying is not considered as 'bridge, 'nexus,' or 'tie' from $\mathrm{t}_{1}$ to $\mathrm{z}$ (or from $\mathrm{t}_{2}$ to $\mathrm{z}$ ), since $\mathrm{t}_{1} \neq \mathrm{z}$, then $\mathrm{t}_{1}$ and $\mathrm{R}$ apparently cannot have any sort of dealings with one another (such as $\mathrm{t}_{1}$ taking part in the coexemplification of $\mathrm{R}$ ). It appears that in order for $\mathrm{R}$ to be exemplified by $\mathrm{t}_{1}\left(\right.$ and $\left.\mathrm{t}_{2}\right), \mathrm{R}$, which is wholly at $\mathrm{z}$ (in the platonistic realm), must also be at $t_{1}$ (and $t_{2}$ ), which his to say that atemporal $R$ must be located at temporal locations, and thus must apparently take on characteristics that are self-contradictory. (The contradiction discussed in this paragraph ensues regardless of whether or not exemplification is considered primitive and unanalyzable.)

\section{Conclusion}

If my preceding arguments are sound, there are no relations between non-identical times, and only an indivisible present exists. Some might wonder how we can account for our experience of change, duration, the persistence of objects, and the specious present if nonsimultaneous temporal relations do not exist. If our universe is composed of philosophic atoms, ${ }^{24}$ the replacement of their present arrangements or interactions, or of their states of interaction, ${ }^{25}$ (where at any

\footnotetext{
${ }^{24}$ Since Peter van Inwagen's book Material Beings, philosophic atoms (basic building blocks), such as those discussed by the Presocratic Greeks (Democritus, etc.) are now usually called 'physical simples'. 'mereological simples,' or 'material simples.' There is much current dialogue in the literature on this issue from such philosophers as Merricks (2001), Markosian (1998), Hudson (2001), McDaniel (2003), Zimmerman (1996a, 1996b), and several others.

${ }^{25}$ In this note, I will very briefly explain why I am using the concept of interaction of atoms here. Typically philosophers hold that ordinary composite objects are collections of atoms that are arranged a certain way (for a good example, see T.
} 
present only one durationless arrangement exists or one set of interactions exists, whatever the nature of these interactions) might account for our experience of time. ${ }^{26}$

On this account, time and the experience of duration would be a phenomenological creation as the mind apprehends or represents arrangements or interactions or states of atoms from durationless present to durationless present. ${ }^{27}$ On such an account, the arrangements or interactions of the atoms are endlessly replacing, but where only one arrangement or set of states or interactions exists at each present. In other words, atoms, which only exist in the present, exist in different states at each new present, where only the states of the atoms, rather than the atoms, are replaced. If there are no temporal parts since there cannot be relations between times, it would seem likely that a presentist theory of time that involves a replacing present is a candidate for a theory of time that could replace the current nonpresentist models of time, and which thereby could replace the widely discussed but apparently incorrect A- and B-theories of time. ${ }^{28}$

\author{
Jeffrey Grupp \\ Dept. of Philosophy and Comparative Religion \\ Western Michigan University \\ Kalamazoo, MI 49008, USA \\ jeffgrupp@aol.com
}

Merricks 2003), where atoms exist in a network of relations. The word 'arrangement' denotes the network of relations that the atoms are in. But if the arguments of this paper are applied to matter and space rather than to time (see Grupp, forthcoming), they lead to the conclusion that there are no relations between nonidentical atoms, or between non-identical chunks of matter or space. If this is the case, then we can account for structures in nature, not by holding that structures are networks of interrelated atoms, but rather by something else going on that is responsible for the existence of structures out of atoms. The only other way I can imagine that philosophic atoms give rise to structures in nature is by way of some sort of interaction among philosophic atoms, if the atoms are not interrelated.

${ }^{26}$ I discuss this model of time in another article; see Grupp 2005.

${ }^{27}$ Also see a very interesting passage in Jubien $(1997,157)$ where Jubien comes quite near to describing a replacing present.

${ }^{28}$ I am grateful to an anonymous referee of this journal for very helpful comments on improving this paper. 
References

Armstrong, D. M. 2001. Universals as Attributes. In Metaphysics: Contemporary Readings, ed. by Michael Loux. New York: Routledge.

Armstrong, D.M. 1997. A World of States of Affairs. Cambridge: Cambridge University Press.

Armstrong, D.M. 1989. Universals: An Opinionated Introduction. Boulder: Westview Press.

Campbell, Keith. 1990. Abstract Particulars. Cambridge: Blackwell.

Chisholm, Roderick. 1996. A Realist Theory of Categories. Cambridge: Cambridge University Press.

Clark, B. L.1981. A Calculus of Individuals Based on 'Connection'. Notre Dame Journal of Formal Logic 22:204-218.

Esfeld, Michael. 2003. Do Relations Required Underlying Intrinsic Properties? A Physical Argument for a Metaphysics of Relations. Metaphysica. Vol. 4, No. 1:5-25

Grossmann, Reinhardt. 1992. The Existence of theWorld. New York: Routledge.

Grünbaum, Adolf. 2001a. Zeno's Metrical Paradox of Extension. In Zeno's Paradoxes, ed. by Salmon, Wesley, Indianapolis: Hackett.

Grünbaum, Adolf. 2001b. Modern Science and Refutation of the Paradoxes of Zeno. In Zeno's Paradoxes, ed. by Salmon, Wesley, Indianapolis: Hackett.

Grünbaum, Adolf. 1952. A Consistent Conception of the Extended Linear Continuum As An Aggregate of Unextended Elements. Philosophy of Science XIX:288-306.

Grupp, Jeffrey. 2005. The R-Theory of Time, or Replacement Presentism: The Buddhist Philosophy of Time. Indian International Journal of Buddhist Studies forthcoming.

Grupp, Jeffrey. forthcoming. The Impossibility of Relations Between NonCollocated Spatial Objects and Non-Identical Topological Spaces. Axiomathes. Forthcoming (in press). (This paper can be read at www.abstractatom.com.)

Grupp, Jeffrey. 2003. The Impossibility of an Exemplification Tie Between Particulars and Universals. Metaphysica: The International Journal for Ontology and Metaphysics. Vol. 4, No. 1: 27-38. (This paper can be read at www.abstractatom.com.)

Hudson, Hud. 2001. A Materialist Metaphysics of the Human Person. Ithaca: Cornell Univeristy Press.

Jones, Vaughan, and Moscovici, Henri. 1997. Review of Noncommutative Geometry by Alain Connes. Notices of the AMS 44:792-799.

Jubien, Michael. 1997. Contemporary Metaphysics, Blackwell: Malden.

Kane, George. 2000. Supersymmetry. Cambridge: Perseus Publishing.

Lesniewski, Andrew. 1997. Noncommutative Geometry. Notices of the AMS $44: 800-805$ 
Loux, Michael. 1998. Metaphysics: A Contemporary Introduction. New York: Routledge.

Lowe, E.J. 2002. A Survey of Metaphysics. New York: Oxford University Press. Madore, J. 2000. An Introduction to Noncommutative Geometry and its Physical Applications, second edition. Cambridge: Cambridge University Press.

Markosian, Ned. 1998. Simples. Australasian Journal of Philosophy 76:213-26.

McDaniel, Kris. 2002. Extended Simples, Shape, and Space. $2^{\text {nd }}$ Annual Princeton-Rutgers Graduate Student Philosophy Conference.

McDaniel, Kris. 2003. Against MaxCon Simples. Australasian Journal of Philosophy 81:265-275.

Mellor, D. H. 1991. Properties and Predicates. In Matters of Metaphysics. Cambridge: Cambridge University Press.

Mellor, D. H. 1992. There Are No Conjunctive Universals. Analysis 52:97-105.

Merricks, Trenton. 2001. Objects and Persons. New York: Oxford University Press.

Moreland, J. P. 2001. Universals. Montreal and Kingston: McGill-Queen's University Press.

Phillips, Stephen H. 1995. Classical Indian Metaphysics. Chicago: Open Court. Pyle, Andrew. 1995. Atomism and Its Critics. Bristol: Thoemmes Press.

Smith, Quentin. 1995. Time, Change, and Freedom. New York: Routledge.

Smith, Quentin. 1993. Language and Time. New York: Oxford University Press.

Vallicella, William. 2000. Three Conceptions of States of Affairs. Nous, 34, 2:237-259.

van Inwagen, Peter. 1990. Material Beings. Cornell University Press: Ithaca.

Wolterstrorff, Nicholas. 1970. On Universals. Chicago: The University of Chicago Press.

Zimmerman, Dean. 1996a. Indivisible Parts and Extended Objects: Some Philosophical Episodes from Topology's Prehistory. The Monist. 79:148-180.

Zimmerman, Dean. 1996b. Could Extended Objects Be Made Out of Simple Parts? An Argument for 'Atomless Gunk.' Philosophy and Phenomenological Research Vol. LVI, No. 1:1-29. 